\title{
Experimental Phase-Sensitive Cloning
}

John C. Howell ${ }^{1}$, Irfan A. Khan ${ }^{1}$, D. Bouwmeester ${ }^{2}$, and N. P. Bigelow ${ }^{1}$

${ }^{1}$ Department of Physics and Astronomy, University of Rochester, Rochester, NY 14627, USA

${ }^{2}$ Department of Physics, University of California at Santa Barbara, Santa Barbara, CA 93106, USA.

This paper has been withdrawn. 